\title{
Qualitative behavior of a coarse-grain growth model
}

\author{
Pasquale Palumbo, Federico Papa \\ Istituto di Analisi dei Sistemi ed Informatica "A. Ruberti" \\ National Research Council (IASI-CNR) \\ Via dei Taurini 19, Rome, Italy \\ \{pasquale.palumbo,federico.papa\}@iasi.cnr.it
}

\author{
Marco Vanoni, Lilia Alberghina \\ Department of Biotechnologies and Biosciences \\ University of Milano-Bicocca \\ Piazza della Scienza 2, Milan, Italy \\ \{marco.vanoni,lilia.alberghina\}@unimib.it
}

\begin{abstract}
The construction of comprehensive, integrative computational models are of exceptional interest in Systems Biology, since they could help researchers to understand the emergent cellular functions and help physicians to treat diseases. Within this framework, detailed molecular models are usually unmanageable because, besides identifiability problems, even correct simulations may not lead to understanding. On the other hand a multi-scaled, modular flexibly-grained modeling approach would provide an overview for the subsequent molecular details, trying to capture the basic emergent properties associated to each module.

This note deals with a minimal model of cellular growth, involving ribosome and protein content. A modified version of the model has been recently implemented as a module of a coarse-grain model combining growth, metabolism and cycle, providing encouraging results for both single cell and population simulations. Here we propose the qualitative behavior analysis of the model, providing model parameters conditions that ensure (or prevent) growth. This way known approximated results are overcome and improved, allowing to infer information about the model qualitative behavior without resorting to numerical simulations.
\end{abstract}

Index Terms-Systems Biology, Qualitative behavior analysis

\section{INTRODUCTION}

Mathematical models describing cell growth and cell cycle events provide a theoretical support for the analysis of the cell life, from a single cell perspective to the cell population level, as well as for the investigation of the mechanisms adopted by the cell to control its size and cycle events (e.g. DNA synthesis and mitosis). In this paper we review the growth model originally published in [2]. Motivation for the proposed mathematical analysis is twofold: from the one hand a slightly modified version of such model has been employed as a module of a coarse-grain model that interconnects three basic cellular functions such as metabolism, growth and cycle [8], paving the way for further whole-cell model developments [9]; from the other hand formal mathematical analysis dates back to the original publication and is constrained to a model approximation that does not include any different growth conditions.

Growth models are of paramount importance because growth affects (and is influenced, in feedback, by) cell cycle progression at many different levels (see, e.g. [1], [3], [7] and references therein). The model here investigated is a minimal

This paper has been supported by a Miur-FOE grant given to ISBE.IT, the Italian node of the ESFRI Research Infrastructure on Systems Biology, to which SYSBIO Centre of Systems Biology belongs. model for ribosome and protein accumulation, the informational macromolecules whose biosynthesis is accounted to drain the large part of energy and building block exploited in cellular processes. Experimental evidence suggests the presence of a negative feedback that reduces the ribosome biosynthesis in presence of ribosomes not engaged in protein biosynthesis, for both bacteria and eukaryotic cells (see, e.g. [4]-[6], [10]). This fact is modeled by means of a switching mechanism that switches off the ribosome production rate any time the ribosomes-over-protein ratio exceeds a given optimal value.

A preliminary mathematical analysis of the qualitative behavior has been carried out in [2], where exponential growth conditions are given according to a specific approximation. Here we extend these results to any feasible setting of the model parameters, without any approximations, showing the mathematical coherence of the model and clearly stating specific growth conditions for any model parameter setting.

\section{THE GROWTH MODEL}

The structure of the growth model proposed in the paper [2] is the same for all cell types (e.g. mammalian cells, yeasts, bacteria), while its parameter setting is cell-dependent. The growth mechanism is represented by means of the two related dynamics of proteins $(P)$ and ribosomes $(R)$. The protein content $P$ is expressed in terms of number of polymerized amino acids and its temporal behaviour depends on the balance between protein synthesis and degradation: the synthesis is given by the product of the number of ribosomes multiplied by their (average) efficiency $K_{2}$ in ammino acids polymerizaton during traslation, while the degradation follows a first-order dynamics with the time constant $\tau_{2}$.

Regards to ribosomes dynamics, a negative feedback is assumed to control the synthesis rate according to the level of ribosomes (as well as of proteins) actually present in the cell. More in details, there is no ribosome synthesis if the ratio $R / P$ overcomes a threshold $\rho$ (i.e., if there are too many ribosomes with respect to the actual quantity of proteins); otherwise, the ribosome synthesis is proportional to the (positive) difference $\rho P(t)-R(t)$, by means of the efficiency $K_{1}$. Such a compensatory mechanism based on the ribosome level is supported by many experimental findings [4]-[6], [10]. The threshold $\rho$ is the ideal ratio of ribosomes over proteins, and it represents the limit value of the actual 
ratio $R / P$ after the transient period. Finally, concerning the ribosome degradation, the clearance rate is assumed trivially linear with a time constant $\tau_{1}$.

Equations for $R(t)$ and $P(t)$ are below reported:

$$
\left\{\begin{array}{l}
\dot{R}(t)=[\rho P(t)-R(t)]^{+}-\frac{R(t)}{\tau_{1}}, \\
\dot{P}(t)=K_{2} R(t)-\frac{1}{\tau_{2}} P(t)
\end{array}\right.
$$

where

$$
[x]^{+}= \begin{cases}x, & \text { for } x>0 \\ 0, & \text { otherwise. }\end{cases}
$$

\section{THE GROWTH MODEL AS A VARIABLE STRUCTURE MODEL}

The Ordinary Differential Equations (ODE) model (1) may be thought of as a variable structure system (aka switching system), with the two linear switching modes triggered by the position of the state of the system in the phase plan. For this reason, in order to characterize the dynamical behaviour of $R(t)$ and $P(t)$, it is worthwhile to study the properties of the two working modes, that is of the two linear dynamical systems included in the compact formulation (1), and to investigate the switching conditions from a working mode to the other one.

The two working modes embedded into the hybrid system (1) are given by the following linear systems:

$$
\begin{gathered}
\mu_{1}: \quad\left\{\begin{array}{l}
\dot{R}(t)=-\frac{R(t)}{\tau_{1}}, \\
\dot{P}(t)=K_{2} R(t)-\frac{P(t)}{\tau_{2}},
\end{array}\right. \\
\mu_{2}: \quad\left\{\begin{array}{l}
\dot{R}(t)=-\left(K_{1}+\frac{1}{\tau_{1}}\right) R(t)+K_{1} \rho P(t), \\
\dot{P}(t)=K_{2} R(t)-\frac{1}{\tau_{2}} P(t) .
\end{array}\right.
\end{gathered}
$$

The working mode $\mu_{1}$, described by system (2), represents a cell that is not synthesizing ribosomes, while the working mode $\mu_{2}$, given by system (3), represents a cell that is actually producing ribosomes. For each working mode it is interesting to establish if cell growth is possible and under which conditions on the model parameters. Let us so denote by $g$ and $\bar{g}$ the two opposite growing states of the cell, that in principle can be obtained under each working mode: the state $g$ represents a growing cell, i.e. the number of ribosomes and proteins is actually increasing; the state $\bar{g}$ represents a not growing cell in which the number of ribosomes and proteins is decreasing.

From the equations of system (2) it is easy to infer that only the growing state $\bar{g}$ (no growth) can be obtained under the working mode $\mu_{1}$ (no ribosome synthesis), meaning that the number of ribosomes and proteins decrease in the absence of ribosome synthesis. Indeed, the eigenvalues of system (2) are both negative, which implies that $R(t) \rightarrow 0, P(t) \rightarrow 0$ for $t \rightarrow \infty$.

Conversely, under the working mode $\mu_{2}$ (ribosome synthesis) both growing states $g$ and $\bar{g}$ are possible. Indeed, the number of ribosomes and proteins can increase or decrease depending on the sign of the related eigenvalues. So, being

$$
A=\left[\begin{array}{cc}
-\left(K_{1}+\frac{1}{\tau_{1}}\right) & K_{1} \rho \\
K_{2} & -\frac{1}{\tau_{2}}
\end{array}\right]
$$

the dynamical matrix of system (3) and

$$
d(\lambda)=\lambda^{2}+\left(K_{1}+\frac{1}{\tau_{1}}+\frac{1}{\tau_{2}}\right) \lambda+\left(K_{1}+\frac{1}{\tau_{1}}\right) \frac{1}{\tau_{2}}-K_{1} K_{2} \rho
$$

the related characteristic polynomial, the eigenvalues of system (3) are given by

$$
\lambda_{1}=y(-1-\sqrt{1+x}), \quad \lambda_{2}=y(-1+\sqrt{1+x}),
$$

where

$$
\begin{gathered}
x=4 \frac{K_{1} K_{2} \rho-\left(K_{1}+\frac{1}{\tau_{1}}\right) \frac{1}{\tau_{2}}}{\left(K_{1}+\frac{1}{\tau_{1}}+\frac{1}{\tau_{2}}\right)^{2}}, \\
y=\frac{1}{2}\left(K_{1}+\frac{1}{\tau_{1}}+\frac{1}{\tau_{2}}\right) .
\end{gathered}
$$

Due to the physical meaning of the model parameters (they are strictly positive), then $y>0$. So, based on the sign of $x$, system (3) gives rise to different dynamical behaviours of $R(t), P(t)$. In particular, from Eqs. (6)-(7) we have that:

- if $x>0$ then system (3) produces the growth of its variables (growth $g$ ). Indeed, $\lambda_{1}, \lambda_{2}$ are real and of opposite sign, that is $\lambda_{1}<0, \lambda_{2}>0$. This means that, after a transient period, both functions $R(t)$ and $P(t)$ tend to an exponential growth with rate constant $\lambda_{2}>0$.

- if $x \leq 0$, no growth of the state variables is produced (no growth $\bar{g}$ ). Indeed, we have:

- if $x=0$ then $\lambda_{2}=0$ while $\lambda_{1}$ is real and strictly negative; so, $R(t)$ and $P(t)$ tend to constant values while the natural mode $e^{\lambda_{1} t}$ approaches zero;

- if $-1 \leq x<0$ then both eigenvalues are real and negative; $R(t)$ and $P(t)$ monotonically converge to zero;

- if $x \leq-1$ then $\lambda_{1}, \lambda_{2}$ are complex conjugate eigenvalues with $\operatorname{Re}\left\{\lambda_{1}\right\}, \operatorname{Re}\left\{\lambda_{2}\right\}<0$; this means that $R(t), P(t)$ converge to zero with oscillations.

Remark 3.1: The linear system described by a non-trivial ribosome synthesis ( $\mu_{2}$ working mode) with a no growth condition $(\bar{g})$ provided by $x \leq-1$ asymptotically converges to zero with oscillations. This means that negative values of both protein and ribosome amounts would be showed. However, such negative, meaningless behavior is prevented by the nonlinear fashion of the whole system (1). Indeed, if there existed a time instant $\bar{t}$ such that $R(\bar{t})=0$ with $\dot{R}(\bar{t})<0$, then by substituting these values in the ribosome dynamics:

$$
\dot{R}(\bar{t})=[\rho P(\bar{t})-R(\bar{t})]^{+}-\frac{R(\bar{t})}{\tau_{1}}=[\rho P(\bar{t})]^{+} \geq 0
$$


which is in contradiction with $\dot{R}(\bar{t})<0$. Analogously it can be proven that $P(t)$ never becomes negative. Therefore, even if starting from a non-trivial ribosome synthesis with $x \leq-1$, there will come a time instant according to which a switch from $\mu_{2}$ to $\mu_{1}$ occurs providing asymptotic convergence to zero without oscillations.

Figure 1 summarizes the combinations of all the possible working modes $\left(\mu_{1}, \mu_{2}\right)$ and growing states $(g, \bar{g})$ of the hybrid system (1), on the basis of the values of the variable ratio $R(t) / P(t)$ and of the quantity $x$. The figure shows that the population of ribosomes and proteins can actually grow only when $x>0$, but it depends on the value of the ratio $R(t) / P(t)$ : the growth state $g$ is actually obtained only under the working mode $\mu_{2}$, i.e. when $R / P<\rho$. Conversely, when $x \leq 0$ the growth is not allowed, independently of the values of the state variables (i.e. of the working mode).

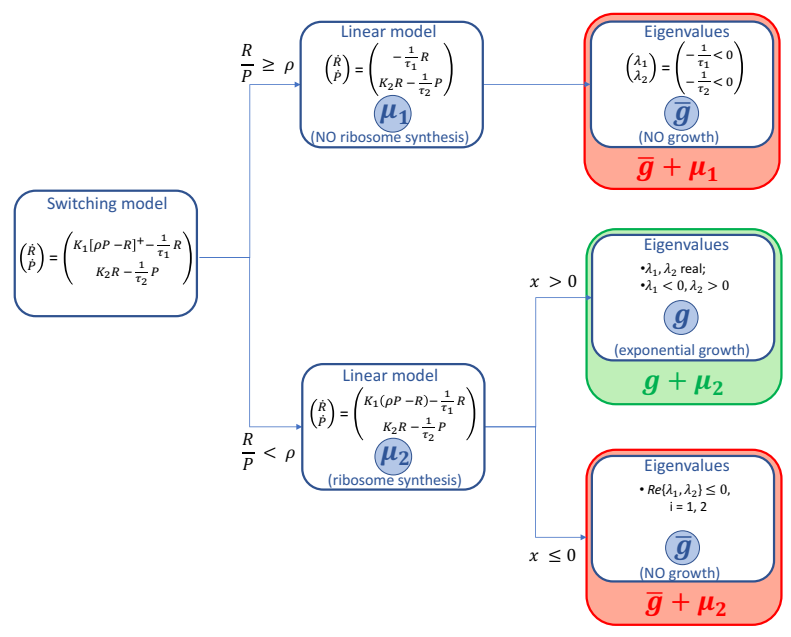

Fig. 1. Combinations of all possible working modes

\section{ANALYSIS OF THE RIBOSOME SYNTHESIS SWITCHES}

The aim of this section is to study when, and under what conditions, the working modes $\mu_{1}$ and $\mu_{2}$ of system (1) remain stable or switch to the other one, on the basis of considerations on the dynamical behaviour of $R(t), P(t)$ (and in particular of their ratio), starting from a particular value $R_{0} / P_{0}$ in $t=0$.

Let us first note from the ODE system (1) that the functions $P(t), R(t)$, and then the ratio $R(t) / P(t)$, are continuous functions with continuous time derivative, for any time $t$ (even at switching times). This means that the time derivative of $R(t) / P(t)$ is actually a continuous function, therefore if the time derivative of $R(t) / P(t)$ is not zero in a given time instant $t$, there exist a small enough neighborhood of $t$ (namely $I_{t}$ ) such that $R(t) / P(t)$ has a monotonic behaviour in $I_{t}$.

\section{A. Switch from $\mu_{1}$ to $\mu_{2}$}

Let us now verify whether the ratio $R(t) / P(t)$ remains larger than, or at least equal to, $\rho$ (no synthesis, $\mu_{1}$ ) or becomes lower than $\rho$ (synthesis, $\mu_{2}$ ), when $R_{0} / P_{0} \geq \rho$ in $t=0$ and $t$ increases from zero. To this end, let us first assume that
$R_{0} / P_{0}>\rho$. Then, because of the continuity of $R(t) / P(t)$, there exists $T>0$ such that $R(t) / P(t)>\rho$ for any $t$ in the interval $[0, T]$, so that the dynamics of $P(t), R(t)$ is given by system (2). Moreover, being

$$
\lambda_{1}=-1 / \tau_{1}<0, \quad \lambda_{2}=-1 / \tau_{2}<0
$$

the eigenvalues of system (2), we note that:

- the ribosome content is explicitly given by the exponential law

$$
R(t)=R_{0} e^{\lambda_{1} t},
$$

that is positive for any $R_{0}>0$ and trivially converges to zero;

- for $\tau_{1} \neq \tau_{2}$ the protein evolution is given by

$$
P(t)=P_{0} e^{\lambda_{2} t}+\frac{K_{2} R_{0}}{\lambda_{1}-\lambda_{2}}\left(e^{\lambda_{1} t}-e^{\lambda_{2} t}\right),
$$

whilst for $\tau_{1}=\tau_{2}$ the protein evolution is given by

$$
P(t)=\left(P_{0}+K_{2} R_{0} t\right) e^{\lambda_{2} t} .
$$

From both Eqs. (12), (13) it follow that $P(t)>0$ for any pair $R_{0}, P_{0}>0$ and $P(t) \mapsto 0$;

- if $\tau_{1} \neq \tau_{2}$, the ratio of ribosomes over proteins is given by:

$$
\begin{aligned}
\frac{R(t)}{P(t)} & =\frac{1}{\frac{P_{0}}{R_{0}} e^{-\alpha t}+\frac{K_{2}}{\alpha}\left(1-e^{-\alpha t}\right)} \\
& =\frac{1}{\left(\frac{P_{0}}{R_{0}}-\frac{K_{2}}{\alpha}\right) e^{-\alpha t}+\frac{K_{2}}{\alpha}}, \quad \alpha=\lambda_{1}-\lambda_{2} ;
\end{aligned}
$$

conversely, if $\tau_{1}=\tau_{2}$ it is

$$
\frac{R(t)}{P(t)}=\frac{1}{\frac{P_{0}}{R_{0}}+K_{2} t} .
$$

Considering first the case $\tau_{1} \neq \tau_{2}$, according to equation (14) we obtain the following results:

- if $\tau_{1}<\tau_{2}$ (i.e. the ribosome clearance rate is faster than the protein one) then $\alpha<0$ and the ratio $R(t) / P(t)$ monotonically decreases to zero. Therefore, there exists a time instant $\bar{t}$ such that $R(\bar{t}) / P(\bar{t})=\rho$. As soon as this happens, the ribosome synthesis will activate, changing the previous working mode (switch from $\mu_{1}$ to $\mu_{2}$ );

- if $\tau_{1}>\tau_{2}$ (i.e. the ribosome clearance rate is slower than the protein one) then $\alpha>0$, from which it follows that the ratio $R(t) / P(t)$ converges to the value $\alpha / K_{2}>0$ (limit value of function (14) for $t \rightarrow \infty$ ). In order to understand whether such convergence occurs in a decreasing or increasing fashion, we compute the time derivative of $R(t) / P(t)$, i.e.

$$
\frac{d}{d t}\left[\frac{R(t)}{P(t)}\right]=\frac{\alpha \frac{P_{0}}{R_{0}}-K_{2}}{\left(\left(\frac{P_{0}}{R_{0}}-\frac{K_{2}}{\alpha}\right) e^{-\alpha t}+\frac{K_{2}}{\alpha}\right)^{2}} e^{-\alpha t},
$$


which is a monotonic function with the sign depending on the initial condition (if positive, it is a positive decreasing function, otherwise it is a negative increasing function). So, based on the sign of $R_{0} / P_{0}$ (and then on the sign of the time derivative of $R(t) / P(t)$ ) we have that:

- if $\alpha / K_{2}>R_{0} / P_{0}>\rho$ then the ratio $R(t) / P(t)$ is monotonically increasing and converges to the limit $\alpha / K_{2}$. So, it will never reach the value of $\rho$ and both $R(t)$ and $P(t)$ will definitely converge to zero;

- if $R_{0} / P_{0}>\alpha / K_{2} \geq \rho$ then the ratio $R(t) / P(t)$ is monotonically decreasing to $\alpha / K_{2} \geq \rho$. Therefore, also in this case $R(t) / P(t)$ will never fall below the threshold $\rho$ and both $R(t)$ and $P(t)$ will definitely converge to zero;

- if $R_{0} / P_{0}=\alpha / K_{2}>\rho$ then the ratio $R(t) / P(t)$ is constant and equal to $\alpha / K_{2}>\rho$, that again prevents the ratio to cross the theshold;

- if $R_{0} / P_{0}>\rho>\alpha / K_{2}$ then the ratio $R(t) / P(t)$ is monotonically decreasing to $\alpha / K_{2}<\rho$. Therefore there exists a time instant $\bar{t}$ such that $R(\bar{t}) / P(\bar{t})=$ $\rho$ according to which the ribosome synthesis will activate, changing the working mode from $\mu_{1}$ to $\mu_{2}$.

From the above discussion we can summarize the result holding for $\tau_{1}>\tau_{2}$, which is actually independent of the initial condition $R_{0} / P_{0}$, as follows:

- if $\rho K_{2} \leq 1 / \tau_{2}-1 / \tau_{1}$ then the ratio $R(t) / P(t)>\rho$, $\forall t>0$; this means there is no switch (stable working mode $\mu_{1}$ );

- if $\rho K_{2}>1 / \tau_{2}-1 / \tau_{1}$ then the ratio $R(t) / P(t)$ sooner or later will go under the threshold $\rho$, switching the working mode from $\mu_{1}$ to $\mu_{2}$.

Regards to the case $\tau_{1}=\tau_{2}$, we have that $R(t) / P(t)$ monotonically decreases to zero (see Eq. (15)), guaranteeing the crossing of the threshold and so the switching of the working mode from $\mu_{1}$ to $\mu_{2}$.

The previous results given for $R_{0} / P_{0}>\rho$ are also valid in case of $R_{0} / P_{0}=\rho$ : it is sufficient to evaluate the sign of the time derivative of the ratio (14), for $\tau_{1} \neq \tau_{2}$, and of the ratio (28), for $\tau_{1}=\tau_{2}$, when the equality $R_{0} / P_{0}=\rho$ holds.

We are now able to provide the following result.

Proposition 4.1: Given the initial time $t_{0}$, with the initial condition $R\left(t_{0}\right) / P\left(t_{0}\right) \geq \rho$, the ribosome synthesis of system (1) is not active and the working mode $\mu_{1}$ is going to change or not for $t>0$, only depending on the model parameters. In particular,

1) the working mode $\mu_{1}$ sooner or later will switch to $\mu_{2}$, if

$$
\tau_{1} \leq \tau_{2}
$$

or if

$$
\tau_{1}>\tau_{2} \quad A N D \quad \rho K_{2}>1 / \tau_{2}-1 / \tau_{1}
$$

2) otherwise the working mode $\mu_{1}$ will not change.
Remark 4.1: It worths noticing that, in case condition $x>0$ holds, then also $\rho K_{2}>1 / \tau_{2}-1 / \tau_{1}$ holds true, since:

$$
x>0 \Longrightarrow \rho K_{2}>\left(1+\frac{1}{K_{1} \tau_{1}}\right) \cdot \frac{1}{\tau_{2}}>\frac{1}{\tau_{2}}-\frac{1}{\tau_{1}}
$$

As a matter of fact, in case $x>0$ holds true, then condition 1) of Proposition 4.1 is always valid, and the switch from $\mu_{1}$ to $\mu_{2}$ will occur for a given time instant.

\section{B. Switch from $\mu_{2}$ to $\mu_{1}$}

Regards to the switch from $\mu_{2}$ to $\mu_{1}$, we limit the study to the case $x>0$ (i.e. when growth is ensured by the working mode $\mu_{2}$ ). Indeed, in such a case the switch of the working mode would produce a nontrivial switch of the qualitative behavior changing from growing to not growing (i.e. $g \rightarrow \bar{g}$ ). In summary, it is interesting to investigate for the case $x>0$, if (and under what conditions) the transition $\mu_{2}+g \rightarrow \mu_{1}+\bar{g}$ can occur.

Let us assume that the model parameters are s.t. $x>0$ (i.e. $\left.K_{1} K_{2} \rho>\left(K_{1}+1 / \tau_{1}\right) / \tau_{2}\right)$ and that $R_{0} / P_{0}<\rho$ in $t=0$. In order to study the behaviour of the ratio $R(t) / P(t)$ when $t$ increases from zero (i.e. if it remains under or crosses the threshold $\rho$ ), we need to compute the explicit solutions of system (3). Such solutions can be given as linear combinations of the natural modes $e^{\lambda_{i} t}, i=1,2$, where the eigenvalues $\lambda_{i}$ are provided by Eqs. (6)-(7). In particular, we have:

$$
\left[\begin{array}{l}
R(t) \\
P(t)
\end{array}\right]=u_{1} v_{1}^{T}\left[\begin{array}{l}
R_{0} \\
P_{0}
\end{array}\right] e^{\lambda_{1} t}+u_{2} v_{2}^{T}\left[\begin{array}{c}
R_{0} \\
P_{0}
\end{array}\right] e^{\lambda_{2} t},
$$

where $u_{i}$ and $v_{i}$ are respectively the right and the left eigenvectors associated to the eigenvalues $\lambda_{i}, i=1,2$ (i.e. the solutions of the systems $\left.\left(A-\lambda_{i} I\right) u_{i}=0, v_{i}^{T}\left(A-\lambda_{i} I\right)=0, i=1,2\right)$. In particular, recalling the expression of the dynamical matrix $A$ in (4), we obtain the following matrices of eigenvectors

$$
\begin{gathered}
U=\left[\begin{array}{ll}
u_{1} & u_{2}
\end{array}\right]=\left[\begin{array}{cc}
\frac{\lambda_{1}+\frac{1}{\tau_{2}}}{K_{2}} & \frac{\lambda_{2}+\frac{1}{\tau_{2}}}{K_{2}} \\
1 & 1
\end{array}\right], \\
V^{T}=\left[\begin{array}{c}
v_{1}^{T} \\
v_{2}^{T}
\end{array}\right]=\left[\begin{array}{cc}
\frac{K_{2}}{\lambda_{1}-\lambda_{2}} & \frac{\lambda_{2}+\frac{1}{\tau_{2}}}{\lambda_{2}-\lambda_{1}} \\
\frac{K_{2}}{\lambda_{2}-\lambda_{1}} & \frac{\lambda_{1}+\frac{1}{\tau_{2}}}{\lambda_{1}-\lambda_{2}}
\end{array}\right] .
\end{gathered}
$$

Then, from Eqs. (20)-(22), we obtain:

$$
\begin{aligned}
R(t)=\frac{\lambda_{1}+\frac{1}{\tau_{2}}}{\lambda_{2}-\lambda_{1}}(- & \left.R_{0}+\frac{\lambda_{2}+\frac{1}{\tau_{2}}}{K_{2}} P_{0}\right) e^{\lambda_{1} t} \\
& +\frac{\lambda_{2}+\frac{1}{\tau_{2}}}{\lambda_{2}-\lambda_{1}}\left(R_{0}-\frac{\lambda_{1}+\frac{1}{\tau_{2}}}{K_{2}} P_{0}\right) e^{\lambda_{2} t} \\
P(t)=\frac{K_{2}}{\lambda_{2}-\lambda_{1}}(- & \left.R_{0}+\frac{\lambda_{2}+\frac{1}{\tau_{2}}}{K_{2}} P_{0}\right) e^{\lambda_{1} t} \\
& +\frac{K_{2}}{\lambda_{2}-\lambda_{1}}\left(R_{0}-\frac{\lambda_{1}+\frac{1}{\tau_{2}}}{K_{2}} P_{0}\right) e^{\lambda_{2} t}
\end{aligned}
$$


Recalling the eigenvalue expressions (6)-(7), it is easy to verify that the following inequalities hold under the growth condition $x>0$ :

$$
\begin{aligned}
& \lambda_{2}-\lambda_{1}=2 y \sqrt{1+x}>0, \\
& \lambda_{1}+\frac{1}{\tau_{2}}<0, \quad \lambda_{2}+\frac{1}{\tau_{2}}>0, \\
& e^{\lambda_{1} t}<1, \quad \forall t>0, \quad e^{\lambda_{2} t}>1, \quad \forall t>0 .
\end{aligned}
$$

Inequalities (25) can be exploited to verify that the explicit solutions of $R(t)$ and $P(t)$ given by Eqs. (23)-(24) are strictly positive for any pair $R_{0}, P_{0}>0$, and that, after the stable mode associated to $\lambda_{1}$ is over, their regime evolution is

$$
\begin{aligned}
& R(t)=\frac{\lambda_{2}+\frac{1}{\tau_{2}}}{\lambda_{2}-\lambda_{1}}\left(R_{0}-\frac{\lambda_{1}+\frac{1}{\tau_{2}}}{K_{2}} P_{0}\right) e^{\lambda_{2} t}>0, \\
& P(t)=\frac{K_{2}}{\lambda_{2}-\lambda_{1}}\left(R_{0}-\frac{\lambda_{1}+\frac{1}{\tau_{2}}}{K_{2}} P_{0}\right) e^{\lambda_{2} t}>0 ;
\end{aligned}
$$

This implies that the limit of the ratio $R(t) / P(t)$ is given by the following expression

$$
\lim _{t \rightarrow \infty} \frac{R(t)}{P(t)}=\frac{\lambda_{2}+\frac{1}{\tau_{2}}}{K_{2}} \triangleq \gamma_{2}>0 .
$$

It can be verified that the growth condition $x>0$ is sufficient to guarantee $\gamma_{2}<\rho$. Thus, in order to prove that $R(t) / P(t)<\rho$ holds for any $t>0$, it is sufficient to prove the monotonic behaviour of $R(t) / P(t)$ by studying the sign of its time derivative. Denoting by $\gamma_{1} \triangleq-\left(\lambda_{1}+1 / \tau_{2}\right) / K_{2}$ and recalling the expressions of $R(t)$ and $P(t)$ given by (23)-(24), the ratio $R(t) / P(t)$ can be written as

$$
\frac{R(t)}{P(t)}=\frac{\gamma_{1}\left(R_{0}-\gamma_{2} P_{0}\right) e^{\lambda_{1} t}+\gamma_{2}\left(R_{0}+\gamma_{1} P_{0}\right) e^{\lambda_{2} t}}{-\left(R_{0}-\gamma_{2} P_{0}\right) e^{\lambda_{1} t}+\left(R_{0}+\gamma_{1} P_{0}\right) e^{\lambda_{2} t}},
$$

from which, computing the time derivative, we obtain

$$
\begin{aligned}
\frac{d}{d t}\left[\frac{R(t)}{P(t)}\right] & \frac{P_{0}^{2}}{K_{2}}\left(\gamma_{2}-\frac{R_{0}}{P_{0}}\right)\left(\frac{R_{0}}{P_{0}}+\gamma_{1}\right)\left(\lambda_{2}-\lambda_{1}\right)^{2} \\
= & \frac{\left.\left(R_{0}-\gamma_{2} P_{0}\right) e^{\lambda_{1} t}+\left(R_{0}+\gamma_{1} P_{0}\right) e^{\lambda_{2} t}\right)^{2}}{\left(\lambda_{1}+\lambda_{2}\right) t},
\end{aligned}
$$

which is a monotonic function with the sign depending on the initial condition $R_{0} / P_{0}$. Indeed, from Eqs. (6)-(7) and the condition $x>0$, we have the inequalities

$$
\lambda_{1}+\lambda_{2}=-2 y<0, \quad \gamma_{1}=-\frac{\left(\lambda_{1}+\frac{1}{\tau_{2}}\right)}{K_{2}}>0,
$$

which imply that the function $e^{\left(\lambda_{1}+\lambda_{2}\right) t}$ is monotonically decreasing $\forall t>0$ and that the sign of (29) only depends on the factor $\gamma_{2}-R_{0} / P_{0}$. In any case, such a sign cannot change with time and the monotonic behaviour of $R(t) / P(t)$ is guaranteed for any value of the initial ratio $R_{0} / P_{0}$. So, we can conclude that $R(t) / P(t)<\rho, \forall t>0$, when $R_{0} / P_{0}<0$. In other words, when the parameter setting is s.t. $x>0$ and the ribosome synthesis is active (working mode $\mu_{2}+$ growing state $g$ ) the transition $\mu_{2}+g \rightarrow \mu_{1}+\bar{g}$ is forbidden.

From the arguments above we provide the following result.

Theorem 4.1: Given the initial time $t_{0}$ and the initial condition $R\left(t_{0}\right) / P\left(t_{0}\right)<\rho$ with $x>0$ (growth condition), the ribosome synthesis of system (1) is active and the state variables show growing limit trends. Such working mode and is maintained for any time.

Figure 2 represents, in the parameter space, all the possible working modes and growing states of system (1), showing when the transitions are forbidden or unavoidable (the transition $\mu_{2}+\bar{g} \rightarrow \mu_{1}+\bar{g}$ is not detailed since no change in the growing condition is implied).

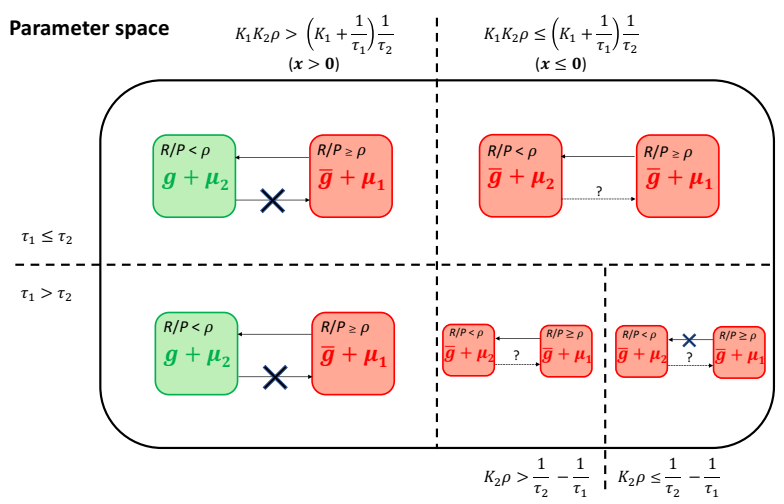

Fig. 2. Switching of the working modes

\section{Conclusions}

The coherence of a mathematical model (i.e. whether the associated solutions are meaningful for the largest range of the feasible model parameters) is an important feature a good model is required to attain, especially when aiming at describing a wide range of possible working modes. In this work we have investigated the qualitative behavior of a coarse-grain model of cellular growth, recently exploited as a module of a larger interconnected model that integrates metabolism, growth and cycle. More in details, we find a specific, sufficient condition $(x>0)$ for the growth of ribosome and protein populations. In particular, when starting the dynamic evolution with active ribosome synthesis such condition guarantees to maintain synthesis and growth for any time. Conversely, when starting with the ribosome synthesis initially inactive, determining a temporary non-growing state, the system approaches a state condition that allows the switch for an active synthesis and, consequently, exponential growth. On the other hands, if such condition is violated $(x \leq 0)$, no growth is possible, independently of the ribosome synthesis.

\section{REFERENCES}

[1] L. Alberghina, et al., "Cell growth and cell cycle in Saccharomyces cerevisiae: basic regulatory design and protein-protein interaction network," Biotechnol. Adv., vol. 30, pp. 52-72, 2012.

[2] L. Alberghina, L. Mariani and E. Martegani, "Cell cycle modelling," Biosystems, vol. 19, pp. 23-44, 1986. 
[3] M. Björklund, "Cell size homeostasis: Metabolic control of growth and cell division," BBA Molecular Research, vol. 1866, pp. 409-417, 2019.

[4] E. Bosdriesz, D. Molenaar, B. Teusink, and F.J. Bruggeman, "How fast-growing bacteria robustly tune their ribosome concentration to approximate growth-rate maximization," FEBS J., vol. 282, pp. 20292044, 2015.

[5] E. Martegano, L. Popolo, L. Alberghina, and E. Sturani, "Reduction of ribosome activity and synthesis of stable RNA in Neurospora crassa," Biochem. Biophys. Acta, vol. 610, pp. 318-330, 1980.

[6] M. Nomura, R. Gourse, and G. Baughman, "Regulation of synthesis of ribosomes and ribosomal components," Ann. Rev. Biochem., vol. 53, pp. $75-117,1984$.

[7] P. Palumbo, M. Vanoni, V. Cusimano, S. Busti, F. Marano, C. Manes, L. Alberghina, "Whi5 phosphorylation embedded in the G1/S network dynamically controls critical cell size and cell fate," Nat. Commun., vol. 7:11372, 2016.

[8] P. Palumbo, M. Vanoni, F. Papa, S. Busti, M. Wortel, B. Teusink, and L. Alberghina, "An integrated model quantitatively describing metabolism, growth and cell cycle in budding yeast," in Pelillo M. et al. (Eds.) Artificial Life and Evolutionary Computation. WIVACE 2017. Communications in Computer and Information Science, Vol. 830. Springer, Cham.

[9] B. Szigeti, Y.D. Roth, J.A.P. Sekar, A.P. Goldberg, S.C. Pochiraju, and J. Karr, "A blueprint for human whole-cell modeling," Current Opinion in Systems Biology, vol. 7, pp. 8-15, 2017.

[10] J.J. Turner, J.C. Ewald, and J.M. Skotheim, "Cell size control in yeast," Curr. Biol., vol. 22, pp. 350-359, 2012. 